\title{
Walk the Line: From Diffusion Imaging to the Microstructure of the Brain
}

\author{
I. Mader $\cdot$ H. Urbach
}

Received: 17 October 2013 / Published online: 13 November 2013

(C) Springer-Verlag Berlin Heidelberg 2013

Diffusion-weighted imaging taught us in the 1990s to identify acute stroke on diffusion-weighted images as diffusion restriction being attributed to pathophysiological changes of the underlying microstructure such as intracellular edema [1-4]. At the same time, the anisotropic orientation of water diffusion being also dependent on the microstructure of the brain was addressed [5] and expressed as self-diffusion tensor [6]. Soon, several tracking methods for fiber tracking were proposed (for a review, see [7]). The most famous and commonly used tracking algorithms are fiber assignment by continuous tracking (FACT) [8-10] and probabilistic tracking [11-15]. Especially FACT algorithm is widely used and has been implemented in commercial neurosurgical planning devices. Why now adding a new tracking method such as global tracking [16-17] to so many others? They all have their disadvantages and limitations. Where is the truth - the ground truth? Global tracking won the award of the "MICCAI 2009 Fiber Cup: Reconstructing Fibers from the Phantom Data." It was the method that reconstructed best the ground truth of a phantom [18] with given fiber bendings and crossings and different anisotropies. How does it perform in the living and diseased brain? This topic is addressed in this issue entitled as "Global Tracking in human gliomas: a comparison with established tracking methods" by Nguyen Thanh et al. The advantage of global tracking is the resolution of fiber crossings and the continuation of the fibers distally to it. On the other hand, the adaptation to the globally estimated fiber tracts leads to a certain rigidity of the reconstruction, so that some strongly bended fibers are not fully accounted for. In this case, probabilistic

H. Urbach $(\bowtie) \cdot$ I. Mader

Dept. of Neuroradiology, Medical Center University of Freiburg,

Freiburg, Germany

e-mail: horst.urbach@uniklinik-freiburg.de tracking is superior. For the clinician, this means that one has to select the appropriate method for each problem, in other words, "to walk the line" — not only along the fibers.

Diffusion kurtosis Imaging is one of the recent developments of the past decade in diffusion tensor imaging [19]. It is based on the measurement of different $b$-values and allows the assessment of the diffusion-weighted signal that has statistically a non-Gaussian distribution (kurtosis). In this signal, different contributions from intracellular and extracellular space and bound and free water (compartments being not necessarily restricted to anatomical borders) are mirrored. This means that a higher kurtosis is associated with higher diffusion heterogeneity and a lower kurtosis with reduced diffusion heterogeneity, such as restricted diffusion. By this means it is possible to find more subtle changes of the integrity of white matter than by the diffusion metrics derived from the Gaussian distribution such as fractional anisotropy and mean, axial and radial diffusivity alone. In this issue, Zhang, Yang, and coworkers reported on the use of diffusion kurtosis imaging in 15 patients with presumably genetic (formerly called idiopathic) epilepsies. Compared with controls, mean kurtosis differed in the white matter of both frontal lobes, and in the gray and white matter of the right parietal lobe. Even if we do not know what it exactly means, it is another hint for an altered microstructure in a diseased brain.

In any case, more information is added for the evaluation of the brain's microstructure and the specific damages being not seen on conventional MR images. The selection of appropriate measurements and theoretically and practically correct evaluation tools is crucial to approach the histological truth of the pathologies. So we also have to "walk the line" for an adequate research in neuroradiology. 


\section{References}

1. Benveniste H, Heldlun LW, Johnson GA. Mechanism of detection of acute cerebral ischemia in rats by diffusion weighted magnetic resonance microscopy. Stroke. 1992;23:746-54.

2. Latour LL, Svoboda K, Mitra PP, Sotak CH. Time dependent diffusion of water in a biological model system. Proc Natl Acad Sci U S A. 1994;91:1229-33.

3. Norris DG, Niendorf T, Leibfritz D. Healthy and infarcted brain tissues studied at short diffusion times: the origin of apparent restriction and the reduction in apparent diffusion coefficient. NMR Biomed. 1994;7:304-10.

4. Moseley ME, Butts K, Yenari MA, Marks M, de Crespigny A. Clinical aspects of DWI. NMR Biomed. 1995;8:387-96.

5. Moseley ME, Cohen Y, Kucharczyk J, Mintorovitch J, Asgari HS, Wendland MF, et al. Diffusion-weighted MR imaging of anisotropic water diffusion in cat central nervous system. Radiology. 1990;176:439-45.

6. Basser PJ, Mattiello J, LeBihan D. Estimation of the effective self-diffusion tensor from the NMR spin echo. J Magn Reson B. 1994;103:247-54.

7. Mori S, van Zijl PC. Fiber tracking: principles and strategies-a technical review. NMR Biomed. 2002 Nov-Dec;15(7-8):468-80.

8. Basser PJ, Pajevic S, Pierpaoli C, Duda J, Aldroubi A. In vivo fiber tractography using DT-MRI data. Magn Reson Med. 2000;44:625-32.

9. Conturo TE, Lori NF, Cull TS, Akbudak E, Snyder AZ, Shimony JS, et al. Tracking neuronal fiber pathways in the living human brain. Proc Natl Acad Sci U S A. 1999;96:10422-7.

10. Mori S, Crain BJ, Chacko VP, van Zijl PC. Three-dimensional tracking of axonal projections in the brain by magnetic resonance imaging. Ann Neurol. 1999;45:265-9.

11. Behrens TE, Woolrich MW, Jenkinson M, Johansen-Berg $H$, Nunes RG, Clare S, et al. Characterization and propagation of uncertainty in diffusion-weighted MR imaging. Magn Reson Med. 2003;50:1077-88.
12. Behrens TE, Berg HJ, Jbabdi S, Rushworth MF, Woolrich MW. Probabilistic diffusion tractography with multiple fibre orientations: What can we gain? Neuroimage. 2007;34:144-55.

13. Hagmann P, Thiran JP, Jonasson L, Vandergheynst P, Clarke $\mathrm{S}$, Maeder P, et al. DTI mapping of human brain connectivity: statistical fibre tracking and virtual dissection. Neuroimage. 2003:19:545-54.

14. Parker GJ, Alexander DC. Probabilistic Monte Carlo based mapping of cerebral connections utilising whole-brain crossing fibre information. Inf Process Med Imaging. 2003;18:684-95.

15. Parker GJ, Haroon HA, Wheeler-Kingshott CA. A framework for a streamline-based probabilistic index of connectivity (PICo) using a structural interpretation of MRI diffusion measurements. J Magn Reson Imaging. 2003;18:242-54.

16. Kreher BW, Mader I, Kiselev VG. Gibbs tracking: a novel approach for the reconstruction of neuronal pathways. Magn Reson Med. 2008;60:953-63.

17. Reisert M, Mader I, Anastasopoulos C, Weigel M, Schnell S, Kiselev V. Global fiber reconstruction becomes practical. Neuroimage. 2011;54:955-62.

18. Fillard P, Descoteaux M, Goh A, Gouttard S, Jeurissen B, Malcolm J, et al. Quantitative evaluation of 10 tractography algorithms on a realistic diffusion $\mathrm{mr}$ phantom. Neuroimage. 2011;56:220-34.

19. Jensen JH, Helpern JA, Ramani A, Lu H, Kaczynski K. Diffusional kurtosis imaging: the quantification of non-gaussian water diffusion by means of magnetic resonance imaging. Magn Reson Med. 2005;53:1432-40. 